\title{
PERFORMANCE COMPARISION OF MFSK FREQUENCY-HOPPED MULTIPLE ACCESS SYSTEMS WITH RTT DECISION OVER A RAYLEIGH FADING CHANNEL
}

\author{
Ing-Jiunn $\mathrm{Su}$ and Jingshown $\mathrm{Wu}$ \\ Department of Electrical Engineering \\ National Taiwan University \\ Taipei, Taiwan 106, R.O.C.
}

\begin{abstract}
The effectiveness of erasure side information generated by Vterbi's ratio threshold test (RTT) decision for frequency-hopped multiple access (FHMA) systems with $M$-ary frequency shift keying (MFSK) is studied. The performances for $M$ up to 16 are presented. The signaling for $M=4$ is revealed to be the best choice under any circumstances over the Rayleigh fading channel. Compared with binary frequency shift keying (BFSK), the signaling for $M=4$ can offer $30 \%$ and $45 \%$ performance gains in achievable maximum capacity for the synchronous and asynchronous hopping systems with hard decision in the receiver, respectively. With RTT decision, the performance of BFSK systems is improved significantly and the performance gap between the signaling for $M=4$ and $M=2$ can be reduced to $25 \%$ in asynchronous hopping systems. The effect of modulation bandwidth expansion, the variation of signal-to-noise ratio in modulation symbol, and different nature in hit period for both hopping types are discussed.
\end{abstract}

\section{INTRODUCTION}

Spread spectrum multiple access (SSMA) techniques have received increasing interest in commercial wireless communications, due to inherent merits of resisting inevitable multipath signals and hostile interference. Among the SSMA techniques, frequency-hopped multiple access (FHMA) is an attractive candidate for broadband wireless multimedia communications [1]. However, in an FHMA system, the transmitted signal may be interfered by other transmitted signals, known as hit event, due to simultaneous occupancy in the same frequency slot. Such hit events are directly related to the numbers of available frequency slots and simultaneous users in the system. To reduce hit events, raising modulation efficiency to increase the number of frequency slots may be effective in a bandlimited system. On the other hand, the decision scheme in the receiver may affect the system performance as well. In a conventional hard decision receiver, the hit symbols are liable to make error decision and the system performance declines severely. If an erasure side information can be generated to indicate the reliability of received symbols, the system performance can be improved. Veterbi's ratio threshold test (RTT) technique possesses simple and elegant characteristics to generate desirable side information. However, the validation of erasure side information may be affected by the signal-to-noise ratio in received symbol. For $M$-ary frequency shift keying (MFSK) with noncoherent receiving scheme, which is usually adopted for FHMA systems, the effect of modulation bandwidth expansion and the variation in signal-to-noise ratio with different $M$ are studied in this paper. Both synchronous and asynchronous hopping systems with RTT decision over the Rayleigh channel are analyzed. The hard decision system can be treated as a special case in the RTT decision system and its result is presented also.

\section{SYSTEM DESCRIPTION AND ANALYSIS}

For an FHMA system with MFSK signaling, the transmitter hops each $M$-ary modulation symbol to one of available frequency slots according to a predetermined hopping pattern. Under our consideration, the hopping interval (chip) is equal to the modulation symbol duration. The hopped symbols are then transmitted and may be hit by other user signals. The numbers of active users and available frequency slots in the system will govern the probability that hit events occur. To fairly judge the performance of different $M$-ary FSK signaling, we use the number of available frequency slots for BFSK signaling as the benchmark and define a normalized user density, $\lambda$, which is the ratio of the number of users to the number of available frequency slots for BFSK signaling. This term will relate to the hit probability in the following analysis. 


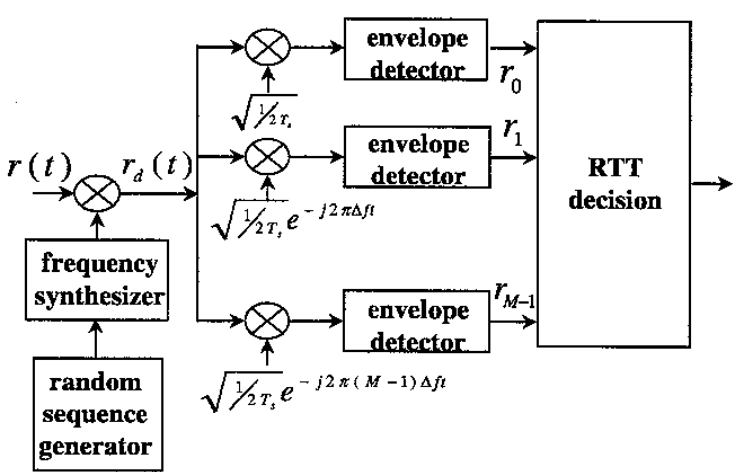

Fig. 1 The receiver structure

As shown in Fig.1, the received signal is dehopped with a desired frequency hopping pattern to strip the hopping effect and then directed to the envelope detectors to generate $M$ decision variables, which can be expressed as $[2],[3]:$

$r_{i}=Z_{i}+C_{i 0} \alpha_{0} e^{j \theta_{0}}+\sum_{m=1}^{K-1} C_{i m} \alpha_{m} x_{m} e^{j \theta_{m}}$

$$
i=0,1, \Lambda M-1
$$

where $K$ is the number of active users in the system, $Z_{i}$ is the complex Gaussian noise with zero mean and variance $\sigma^{2} \equiv N_{0} / 2, N_{0}$ is the one-sided spectral density of additive white Gaussian noise, $C_{i m}$ is the indicator with binary value " 1 " and " 0 " to represent whether the $m$-th user presents signal in the $i$-th decision variable, $\alpha_{m}$ and $\sigma_{m}$ are the amplitude and phase of the $m$-th user signal, and $x_{m}$ is the normalized ratio of hit period to symbol duration for the $m$-th user signal (interference). Here the signal of user 0 is separated from other user signals to represent the intended signal to receive. For synchronous hopping systems, the hit period will be spanned over entire symbol duration if the interference signal collides with the desired signal; therefore, $x_{m}$ would be equal to 1 . On the other hand, the hit period will vary within the symbol duration for asynchronous hopping systems and $x_{m}$ would be an uniform random variable distributed over $[0,1)$ to reflect this partial hit nature. For our model of fading channel, $\theta_{m}$ is an uniformly distributed over $[0,2 \pi)$ and $\left\{\alpha_{m}\right\}$ are the independent and identically distributed (i.i.d.) Rayleigh random variables with variance $2 \sigma_{f}^{2}$ by assuming that all user signals are received with the same average power. If the $M$-ary symbols are equally likely for transmission, we can assume the desired signal is presented in the 0 -th decision variable without loss of generality. Then the corresponding characteristic function of the random variable $r_{i}$ averaging with respective to $\left\{x_{m}\right\}$ can be derived as [4],[5]:

$\phi_{i}(\rho)=e^{-\frac{\left(\sigma^{2}+\delta_{i} 0 \sigma_{f}^{2}\right) \rho^{2}}{2}} \prod_{m=1}^{K-1} \phi_{x}\left(C_{i m} \rho\right)$,

where

$\phi_{x}(\gamma)= \begin{cases}e^{-\frac{\sigma_{f}^{2} \gamma^{2}}{2}}, & \text { synchronous hopping } \\ \int_{0}^{1} e^{-\frac{\sigma_{f}^{2} \gamma^{2} x^{2}}{2}} d x, & \text { asynchronous hopping }\end{cases}$

and $\delta_{i 0}$ is the Kronecker delta. By applying Poisson approximation to average the random variables $\left\{C_{i m}\right\}$ $[2],[5],[6]$, the average characteristic function can be simplified as:

$$
\hat{\phi}_{i}(\rho) \cong\left\{\begin{array}{c}
e^{-\frac{\left(\sigma^{2}+\delta_{i} \sigma_{f}^{2}\right) \rho^{2}}{2}} e^{\frac{\lambda_{M}}{M}\left[\phi_{x}(x)-1\right]}, \\
\text { synchronous hopping } \\
e^{-\frac{\left(\sigma^{2}+\delta_{i} 0 \sigma_{f}^{2}\right) \rho^{2}}{2}} e^{\frac{2 \lambda_{M}}{M}\left[\phi_{x}(x)-1\right]}, \\
\text { asynchronous hopping }
\end{array}\right.
$$

where $\lambda_{M}$ is the user density for MFSK signaling. If the bandwidth expansion effect is taken into account for the bandlimited system, $\lambda_{M}$ can be related to $\lambda$ by the following expression:

$\lambda_{M}=\frac{M}{\log _{2} M} \lambda$

The probability density function (pdf) and cumulative distribution function (cdf) of the random variable $r_{i}$ can be obtained by inverse transformation [7],[8]:

$f_{i}(r)=r \int_{0}^{\infty} \rho J_{0}(r \rho) \hat{\phi}_{i}(\rho) d \rho$

and

$F_{i}(r)=r \int_{0}^{\infty} J_{1}(r \rho) \hat{\phi}_{i}(\rho) d \rho$

where $J_{0}(\bullet)$ and $J_{1}(\bullet)$ are the zeroth and first order Bessel functions, respectively.

With RTT decision scheme, the receiver sets a threshold $(0<t \leq 1)$ to dictate the reliability of received symbol. If the amplitude ratio of the second largest one to the largest one among $M$ decision variables is greater than this threshold, the symbol is declared unreliable and erased; otherwise, the rule used in hard decision would be adopted. Note that no side information will be 


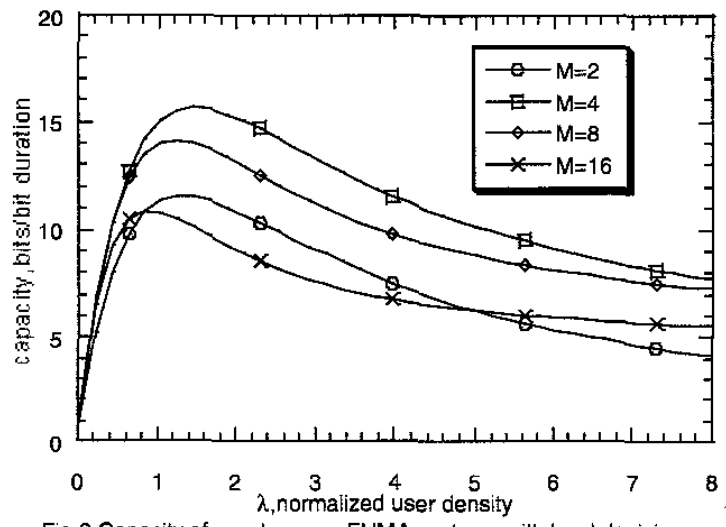

Fig.2 Capacity of synchronous FHMA systems with hard decision generated and the decision can be treated as a hard decision if the threshold is set to 1. For the symbols not being erased, the decision error occurs when the amplitude ratio of $r_{0}$ to $r_{i}$ for $i \neq 0$ is less than $t$ and its average probability can be determined by:

$$
P_{e}(K)=\int_{0}^{\infty} F_{0}(t r) \sum_{i=0}^{M-1} \prod_{j=1, j \neq i}^{M-1} F_{j}(t r) f_{i}(r) d r
$$

Similarly, the average probability of symbol correction can be found as:

$$
P_{c}(K)=\int_{0}^{\infty} \prod_{i=0}^{M-1} F_{i}(t r) f_{0}(r) d r
$$

Finally, the average erasure probability can be obtained by:

$$
P_{x}(K)=1-P_{c}(K)-P_{e}(K)
$$

The total capacity, in terms of bits per channel use, can be computed by summing individual channel capacity [9]:

$$
\begin{aligned}
C(K)=K\left\{P_{c}(K) \log _{2} \frac{M P_{c}(K)}{1-P_{x}(K)}+\right. \\
\left.P_{e}(K) \log _{2} \frac{m P_{e}(K)}{(M-1)\left[1-P_{z}(K)\right]}\right\}
\end{aligned}
$$

The normalized capacity, in terms of bits per bit duration, can be found by dividing the total capacity with $\log _{2} M$ :

$w_{M}=\frac{C(K)}{\log _{2} M}$

\section{NUMERICAL RESULTS}

We first present the performance of the system with synchronous hopping and hard decision schemes. The capacity for various MFSK signaling with

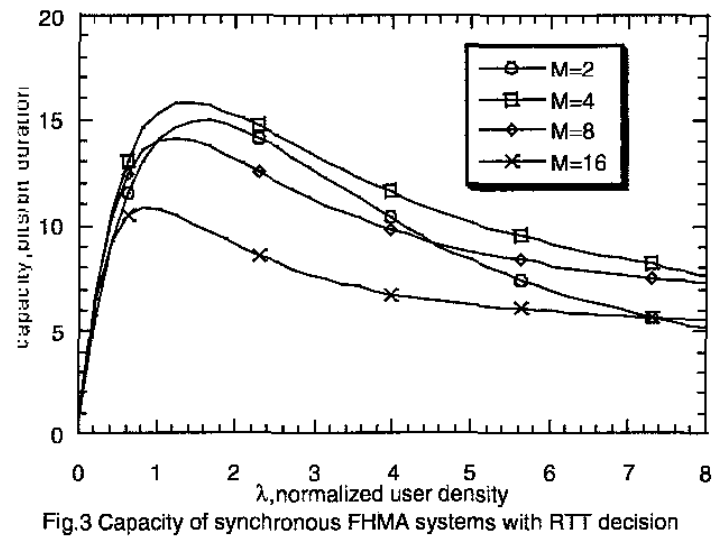

$E_{b} / N_{0}=10 \mathrm{~dB}$ is shown in Fig. 2, where $E_{b}$ is the average bit energy. With the same modulation bandwidth expansion, the signaling for $M=4$ offers more capacity than the binary signaling for all range of user density. The improvement in achievable maximum capacity can be over $30 \%$. In general, the performance declines as $M$ increases, due to reduction in the number of frequency slots. However, the signaling for $M=8$ surpasses the binary signaling in capacity. This fact results from that the higher signal-to-noise ratio in modulation symbol may offset the reduction in the number of frequency slots. The signaling for $M=16$ also exists the similar phenomenon. If the threshold of RTT decision is reduced to generate some erasure side information, the performance may be improved. With the numerical search, we present the optimum performance in Fig. 3. Although the signaling for $M=4$ still has the best performance, the binary signaling may compete with the signaling for $M=4$ or 8 . Moreover, the difference in capacity between the systems with hard decision and RTT decision is insignificant for $M=8$ or 16 . This fact can be explained by that the system with larger $M$ is liable to become an interference-limited system due to reduction in the number of available frequency slots. Since the useful information is reduced by erasure side information, the optimum performance with RTT decision should tend to that with hard decision for an interference-limited system. However, the effectiveness of side information for $M=2$ is prominent for small user density.

As the phenomenon of partial hit in the asynchronous system, the performance behavior may differ from that of the synchronous system. Fig. 4 shows the result with $E_{b} / N_{0}=10 \mathrm{~dB}$ for the systems with hard decision scheme. Evidently, the capacity for the same $M$ in the 


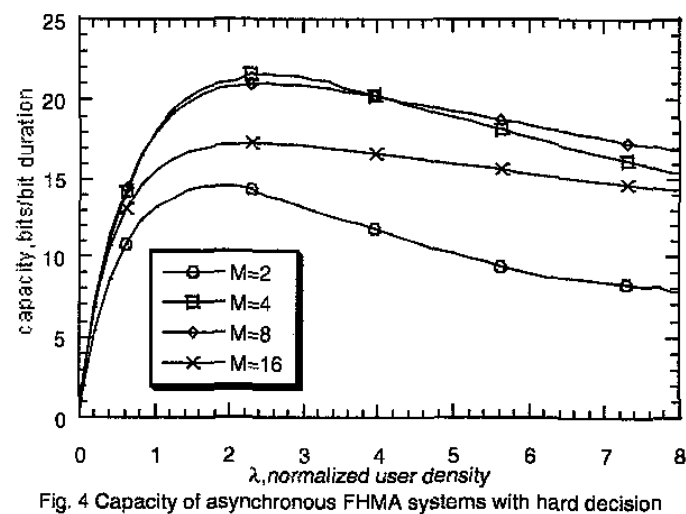

asynchronous system is increased. However, the difference in achievable maximum capacity between the signaling for $M=4$ and $M=2$ is enlarged to $45 \%$. Also we find that the signaling for $M=4$ and 8 are almost best in capacity for moderate user density. The tradeoff between frequency slot number and signal-to-noise ratio appears again and is enforced in the asynchronous system due to partial hit. That can also explain why the binary signaling behaves poor. Happily the RTT decision scheme remedies the system with binary signaling and its optimum performance is competitive for moderate user density, as shown in Fig. 5. The performance gap between the signaling for $M=4$ and $M=2$ is reduced to $25 \%$. The same argument for the difference between hard decision and RTT decision schemes in the synchronous system is also applicable in the asynchronous system.

\section{Conclusion}

The effectiveness of RTT decision for non-coherent MFSK FHMA systems over the Rayleigh fading channel is evaluated in this paper. Both synchronous and asynchronous hopping systems are studied. The conventional hard decision scheme can be treated as a special case of RTT decision. Numerical results reveal that the signaling for $M=4$ performs best in capacity under any circumstance. For the systems with hard decision, the signaling for $M=4$ can offer $30 \%$ and $45 \%$ performance gain in achievable maximum capacity, compared with BFSK signaling in the synchronous and asynchronous cases, respectively. With RTT decision, the performance of BFSK signaling can be improved significantly and compete with the signaling for $M=4$ in synchronous system. For asynchronous case, the performance gain reduces to $25 \%$. Compared with the signaling for $M=4$, the performance declines for larger $M$, due to the effect of bandwidth expansion. With the

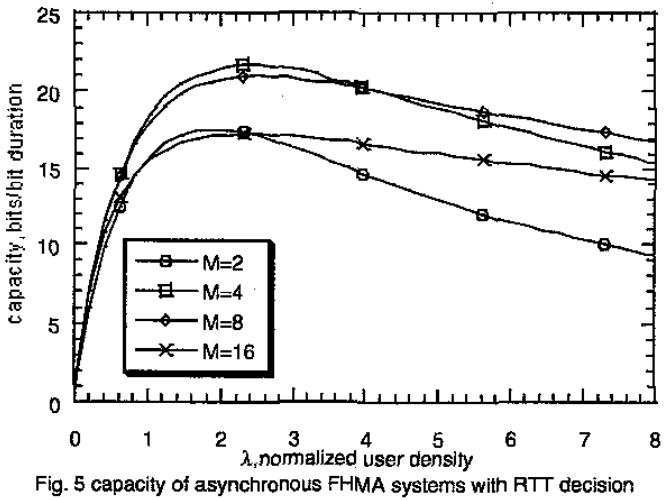

nature of partial hit, the declination in the asynchronous system would be smaller than the one in the synchronous system.

\section{REFERENCES}

[1] N. Morinaga, M. Nskagawa and R. Kohno, "New concepts and technologies for achieving highly reliable and high-capacity multimedia wireless communications systems," IEEE Commun. Mag., pp. 34-40, Jan 1997.

[2] I.-J. Su and J. Wu, "Performance of asynchronous slow-frequency-hopped multiple access systems with RTT techniques for side information," IEICE Trans. Fundamentals of Electronics, Communications and Computer Sciences, vol. E81-A, No. 2, pp. 327-332, Feb. 1998

[3] I.-J. Su and J. Wu, "Capacity of a synchronous BFSK frequency-hopped multiple access system with RTT erasure decision," IEE Electron. Lett., vol. 34, No. 2, pp.144-146, Jan. 1998

[4] C. P. Hung and Y. T. Su, "Diversity combining considerations for incoherent frequency hopping multiple access systems," IEEE J. Select. Areas Commun., vol. 13, pp. 333-344, Feb. 1995.

[5] C. W. Baum and M. B. Pursley, "A decision-theoretic approach to the generation of side information in frequency-hop multiple-access communications," IEEE Trans. Commun., vol. 43, pp. 1768-1777, Apr. 1995.

[6] Ramón Agusti, "On the performance analysis of asynchronous FF-SSMA communications," IEEE Trans. Commun., vol. 37, pp. 488-499, May 1989.

[7] J. S. Bird, "Error performance of Binary NCFSK in the presence of multiple tone interference and system noise," IEEE Trans. Commun., vol. 33, pp. 203-209, Mar. 1985.

[8] J. S. Bird and D. A. George, "The use of the FourierBessel series in calculating error probabilities for digital communication systems," IEEE Trans. Commun., vol. 29, pp. 1357-1365, Sep. 1981. 
[9] K. Cheun and K. Choi, "Performance of FHSS multiple-access networks using MFSK modulation," IEEE Trans. Commun., vol. 44, pp. 1514-1526, Nov. 1996. 